\title{
Apoyo social, autonomía, compromiso académico e intención de abandono en estudiantes universitarios de primer año
}

\author{
Yaranay López-Angulo ${ }^{*}$, Rubia C. Cobo-Rendón ${ }^{2}$, María V. Pérez-Villalobos ${ }^{3}$, y Alejandro E. Díaz-Mujica ${ }^{3}$ \\ (1) Escuela de Psicología, Facultad de Ciencias Sociales y Comunicaciones, Universidad Santo Tomás, Concepción, \\ Chile (correo-e: yara13190@gmail.com). \\ (2) Laboratorio de Investigación e Innovación Educativa, Dirección de Docencia, Universidad de Concepción, \\ Concepción-Chile (correo-e: rubiacobo@udec.cl). \\ (3) Dpto. de Psicología, Facultad de Ciencias Sociales, Universidad de Concepción, Concepción-Chile \\ (correo-e: marperez@udec.cl; adiazm@udec.cl).
}

Recibido Dic. 10, 2020; Aceptado Feb. 8, 2021; Versión final Mar. 9, 2021, Publicado Jun. 2021

\begin{abstract}
Resumen
Este estudio evaluó la capacidad predictiva del apoyo social, apoyo a la autonomía y compromiso académico sobre la intención de abandono de estudiantes universitarios. Se utilizó un diseño explicativo transversal con variables latentes. Participaron 3121 estudiantes (mujeres $=50.1 \%$; hombres $=49.9 \%$; edad promedio $=19.2$ años) de seis universidades chilenas. Se realizaron análisis factoriales confirmatorios y modelos de ecuaciones estructurales para la estimación del modelo explicativo. Los resultados indican que la percepción de apoyo social y apoyo a la autonomía predicen la intención de abandono del estudiante cuando es mediada por el compromiso académico. El apoyo social, autonomía y compromiso explican el $24 \%$ de la intención de abandono. Se concluye que las relaciones entre las variables percepción de apoyo social, percepción de apoyo a la autonomía y compromiso académico, constituyen un modelo explicativo de la intención de abandono en estudiantes universitarios chilenos de primer año.
\end{abstract}

Palabras clave: apoyo social; autonomía; compromiso académico; abandono de cursos; educación superior

\section{Social support, autonomy, academic commitment, and drop out intention in first year undergraduate students}

\begin{abstract}
The primary objective of the present study was to assess the capacity of social support, autonomy support, and academic commitment for predicting undergraduate student drop out intention. A transversal explanatory design with latent variables was used. The participants were 3,121 students (women $=50.1 \%$; men $=49.9 \%$; average age $=19.2$ years) studying at six Chilean universities. Confirmatory factor analyses and structural equation modeling were used to estimate an explanatory model. The results indicate that both social support and autonomy support perceptions predicted student drop out intention when mediated by academic commitment. Social support, autonomy, and commitment explained $24 \%$ of drop out intention. It is concluded that the relationships between perceived social support, perceived support for autonomy, and academic commitment constitute an explicative model for drop out intention of first year Chilean undergraduates.
\end{abstract}




\section{INTRODUCCIÓN}

Durante el ingreso a la universidad, los estudiantes vivencian y necesitan hacer frente a las demandas y exigencias académicas, sociales, y emocionales. Cuando los estudiantes no logran ajustarse al nuevo contexto, se ven mermadas sus capacidades de adaptación, afectando su desempeño y éxito, poniendo en riesgo la permanencia en sus carreras (Phillips et al., 2020; Rodríguez et al., 2017). Debido al impacto que tiene el abandono de los estudios en la vida de los estudiantes, en las instituciones de Educación Superior y en la sociedad, se han identificado diversas variables de tipo personal, académico-educativo, institucionales, económicas y culturales que pueden intervenir en la decisión de continuar o no los estudios universitarios (Gallegos et al., 2018).

La decisión de abandonar los estudios está precedida por la intención de abandono; esta se refiere a pensamientos relativos a cesar la permanencia en un programa formativo conducente a un título de Educación Superior, antes de alcanzar la titulación; se operativiza en verbalizaciones respecto de dejar sus estudios universitarios (Díaz-Mujica et al., 2018) y en comportamientos de menor compromiso académico por parte de los estudiantes. Los pensamientos de abandonar la carrera universitaria y la verbalización de la intención de hacerlo, son frecuentes en mayor medida durante el primer año académico (Crede y Niehorster, 2012; Bernardo et al., 2017). Entre los factores personales que influyen en el abandono de los estudios universitarios, se encuentra el nivel de adaptación a la universidad (Crede y Niehorster, 2012); una revisión metaanalítica de las respuestas de $\mathrm{N}=44.668$ estudiantes universitarios, encontró que la adaptación de los estudiantes está relacionada con aspectos individuales, como: apoyo social y relaciones de los estudiantes con su familia, pares y profesores (Crede y Niehorster, 2012).

Las relaciones sociales, específicamente las percepciones de apoyo social que puede tener el estudiante durante su estancia en la universidad, intervienen de forma favorable en los procesos de adaptación y en el desempeño académico (Rodríguez et al., 2017; Tinajero et al., 2020). Estudiantes que perciben mayor apoyo social, se adaptan mejor a los desafíos que implica la universidad (Bordes-Edgar et al., 2011; Crede y Niehorster, 2012). El apoyo social puede ser definido como la percepción o experiencia de ser atendido por otros y de contar con una red confiable a la cual recurrir cuando se necesita, puede ser entendido como una sensación subjetiva de ser apoyado (Santini et al., 2015). Esta percepción de atención puede ser representada desde diversas experiencias con distintas personas, en tal sentido, las fuentes de apoyo social normalmente provienen de la familia, amistades y otras personas consideradas como significativas (Zimet et al.,1988).

Para un funcionamiento saludable y el incremento del bienestar en las distintas facetas de la vida humana, se necesita cubrir las necesidades psicológicas básicas de autonomía, competencia y relación. Desde la Teoría de la Autodeterminación, existen diversas fuentes de motivación que llevan a las personas a satisfacer estas necesidades. Para esta teoría, la autonomía se refiere a la auto aceptación de las propias capacidades, a decidir sobre las propias conductas y compromisos, corresponde a una capacidad de autodeterminación y su consiguiente comportamiento autorregulado exento de presiones externas (León y Núñez, 2013). En el contexto del aula de clases, cuando los docentes fomentan en sus estudiantes la autonomía, les están facilitando sentimientos de dominio respecto a su aprendizaje, desarrollo de conductas autodeterminadas, les permiten cumplir con las demandas académicas con mayor satisfacción con sus estudios; estas estrategias pueden ser vistas como la posibilidad que los docentes otorgan a los jóvenes para desenvolverse y realizar las actividades académicas con mayor dominio (Hernández y Moreno-Murcia, 2017; Oriol-Granado et al., 2017).

Otra variable relevante en los procesos de adaptación y continuidad de los estudios es el compromiso académico, que puede ser entendido como un estado mental positivo relacionado con el estudio, caracterizado por vigor, dedicación y absorción (Salanova, 2010). El vigor implica poseer altos niveles de energía y agilidad mental, manifestado en comportamientos de persistencia y esfuerzo durante el estudio; la dedicación alude a una profunda involucración en el trabajo académico, entusiasmo, orgullo e inspiración relacionada con los estudios, y finalmente, la absorción corresponde a un estado agradable de concentración en el trabajo, en que el estudiante es incapaz de separarse de la tarea aunque haya transcurrido mucho tiempo (Schaufeli et al., 2006; Schaufeli et al., 2002).

Cuando los estudiantes están comprometidos, conductualmente presentan altos niveles de concentración en el aprendizaje, están emocionalmente interesados en aprender y tienden a involucrarse con sus compañeros de clase y profesores (Noble y McGrath, 2015). También emplean altos niveles de recursos psicológicos, lo que impacta positivamente su desempeño académico (Martínez et al., 2019). El compromiso académico es considerado como un predictor significativo de la intención de abandono temprano de la universidad, esto se debe a que estudiantes con implicación en sus estudios, entusiasmo e inspiración en sus carreras tienen menos probabilidades de tener intención de abandonar (Truta et al., 2018). 
En la Educación Superior, las investigaciones acerca del abandono tradicionalmente han sido analizadas desde la mirada de las universidades, pero debido a la relevancia de factores personales de los estudiantes en la intención de abandono recientemente Tinto (2017), propuso un modelo conceptual de persistencia institucional desde la óptica de los propios estudiantes. Tinto plantea que el estudiante, en este contexto, se ve a sí mismo como miembro de una comunidad, valora la participación en las interacciones diarias con otros y el resultado de este proceso es un vínculo expresado en términos de compromiso. Considerando este planteamiento y las escasas investigaciones empíricas sobre el tema con diseños predictivos y explicativos en Chile, la presente investigación se propuso como objetivo evaluar la capacidad predictiva de la percepción de apoyo social proveniente de fuentes como la familia, los amigos y otras personas significativas, el apoyo a la autonomía y el compromiso académico sobre la intención de abandono de estudiantes universitarios chilenos de primer año.

\section{MÉTODO}

El presente estudio corresponde a un diseño explicativo transversal con variables latentes (Ato et al., 2013). Este tipo de diseño está representado mediante modelos de ecuaciones estructurales; para lo cual se propuso un modelo con estrategia de tipo confirmatoria, buscando verificar el ajuste del modelo hipotetizado a partir de la teoría y de la evidencia empírica (Wang y Wang, 2019. Se propuso un modelo hipotético donde el compromiso académico es una variable mediadora en las relaciones de la percepción de apoyo social y apoyo a la autonomía con la intención de abandono de los estudiantes.

\section{Participantes}

Participaron 3121 estudiantes de primer año de seis universidades chilenas ( 3 privadas y 3 estatales), de las cohortes 2017 y 2018 , de estos 1.558 hombres (49.9\%) y 1.563 mujeres, que tenían en promedio 19.2 años $(D E=1.82)$. El $84.5 \%$ era primera vez que ingresaba a la universidad. El $75.5 \%$ del total, marcó su carrera como primera preferencia al ingresar a la universidad. Los participantes pertenecían en un $40.01 \%$ a carreras de Ingeniería y Tecnología, 6,66\% a Ciencias Naturales, $15.44 \%$ a Ciencias Médicas y de la Salud, $6.34 \%$ a Ciencias Agrícolas, $25.18 \%$ a Ciencias Sociales y $6.344 \%$ a Humanidades, en la tabla 1 se describe la distribución de estudiantes por sexo y área. El muestreo fue no probabilístico accidental.

Tabla 1. Descripción de los participantes según sexo y área de formación científica.

\begin{tabular}{|c|c|c|c|}
\hline \multirow{2}{*}{ Área según la $O C D E$} & \multicolumn{2}{|c|}{ Sexo } & \multirow{2}{*}{ Total } \\
\hline & Masculino & Femenino & \\
\hline Ingeniería y Tecnología & 830 & 419 & 1249 \\
\hline Ciencias Naturales & 135 & 73 & 208 \\
\hline Ciencias Médicas y de la Salud & 151 & 331 & 482 \\
\hline Ciencias Agrícolas & 56 & 142 & 198 \\
\hline Ciencias Sociales & 312 & 474 & 786 \\
\hline Humanidades & 74 & 124 & 198 \\
\hline Total & 1558 & 1563 & 3121 \\
\hline
\end{tabular}

Variables e instrumentos de medición

Se utilizó un cuestionario socio-demográfico para obtener información general de los participantes como edad, sexo, universidad, año de ingreso a la carrera, nombre de la carrera que cursa; además se utilizaron escalas de medición para la evaluación de las variables de estudio, las mismas se describen a continuación. Escala Multidimensional de Apoyo Social Percibido (MSPSS): se utilizó el MSPPS (Zimet et al., 1989), específicamente una versión validada con estudiantes universitarios chilenos (López et al, 2021). Este cuestionario de auto-informe evalúa la percepción de apoyo social que poseen las personas de tres fuentes relevantes (familia, amigos y otros significativos). Se compone por 12 ítems que conforman tres dimensiones: familia (4 ítems) "Tengo la seguridad que mi familia trata de apoyarme"; amigos (4 ítems) "Puedo contar con mis amigos cuando tengo problemas" y otros significativos (4 ítems) "Hay una persona que me ofrece consuelo cuando lo necesito". Niveles altos en estas dimensiones indican mayor percepción de apoyo social. La fiabilidad de las dimensiones fue de $\alpha=.90$ para familia, $\alpha=.93$ para amigos y $\alpha=.86$ para otros significativos. Cuestionario de Clima de Aprendizaje: se utilizó la versión en español de este cuestionario (Matos-Fernández, 2009), que tiene como objetivo evaluar la percepción del estudiante sobre el grado en que el docente 
promueve su autonomía. Un ejemplo de ítems es el siguiente: "Mis profesores me dan opciones y posibilidades de hacer elecciones". Mayores puntuaciones indican mayor percepción de apoyo del estudiante por parte del profesor. Este cuestionario presenta índice de consistencia interna de .93 y reporta una estructura unifactorial. En el presente estudio se encontró un adecuado índice de confiabilidad $\alpha=.89$.

Escala de Compromiso Académico: se utilizó la versión validada de con estudiantes universitarios UWES-9 (Schaufeli et al., 2002). Evalúa el nivel de compromiso del estudiante en el cumplimiento de sus actividades académicas. Se compone por 3 dimensiones: vigor (voluntad para esforzarse y persistir durante el estudio), dedicación (nivel de involucramiento en la actividad académica) y absorción (grado de concentración en la tarea académica). Niveles altos en estas dimensiones indican mayor compromiso en la realización de las actividades académicas. En muestras de estudiantes chilenos este cuestionario ha presentado adecuados niveles de consistencia interna tanto para sus dimensiones (vigor $\alpha=.80$, dedicación $\alpha=.81$ y absorción $\alpha=.72$ ), como para la escala completa $(\alpha=.90)$ (Carmona-Halty et al., 2019). Para este estudio se utilizó la escala completa que presentó un índice de consistencia interna de .90.

Intención de abandono: La intención de abandono fue medida a través de 4 ítems ad hoc, éstos buscaban identificar la presencia de pensamientos asociados al retiro universitario, estos fueron "Espero completar mis estudios en esta carrera", "Deseo continuar estudiando la misma carrera", "Estoy pensando en cambiarme a otra carrera" y "Estoy pensando seriamente en tomarme un tiempo de descanso de la Universidad y continuar después". Se utilizó una escala de respuesta de 7 alternativas (1= muy en desacuerdo a 7= Totalmente de acuerdo). Para calcular la variable, la escala de los dos primeros ítems fue invertida.

\section{Procedimiento}

Se consideraron las normas éticas de investigación con seres humanos en todo el proceso. A todos los estudiantes se les informó sobre los objetivos del estudio y les fue presentado un consentimiento informado que describía las características y condiciones de la investigación. El tiempo de respuesta de los cuestionarios no superó los 20 minutos. Los instrumentos fueron respondidos en las salas de clases de las distintas universidades previo autorización de las autoridades institucionales de mayo a julio de primer semestre de 2019.

El modelo de ecuaciones estructurales propuesto consideró las variables percepción de apoyo a la autonomía y percepción de apoyo social como variables exógenas y a la intención de abandono y compromiso académico como variables endógenas, observándose esta última también como variable mediadora. Además, se consideraron factores de segundo orden para el apoyo social. Se utilizó el método de estimación de Máxima Verosimilitud Robusta (MLR) para la realización del análisis de ecuaciones estructurales y para evaluar la bondad de ajuste se seleccionaron los siguientes índices: raíz cuadrada media de error de aproximación (RMSEA) con un intervalo de confianza al 90 \% (I.C.), el índice de ajuste comparativo (CFI), índice de TuckerLewis (TLI) y la raíz media cuadrática estandarizada (SRMR), definiendo los siguientes criterios: (a) CFI o TLI sobre .90, (b) SRMR menor o igual a .080 con CFI sobre .92, (c) RMSEA menores a .070 con CFI igual o mayor a .90 (Wang y Wang, 2019).

\section{RESULTADOS}

En la tabla 2, se describen los resultados del modelo de medición, donde: df = grados de libertad del modelo; RMSEA = Error Medio Cuadrático de Aproximación; $(90 \% \mathrm{Cl})=$ Intervalo de confianza del $90 \%$ para RMSEA; $\mathrm{TLI}=$ índice de Tucker-Lewis; CFI= Índice de Ajuste Comparativo; ${ }^{*} \mathrm{p}<.0{ }^{* *} \mathrm{p}<.01$. Los resultados confirman la estructura factorial de cada variable analizada, para posteriormente poder incluirla en el análisis de ecuaciones estructurales.

El análisis de la comprobación de los modelos de medida es un paso previo y necesario para el desarrollo de este tipo de modelos. Los resultados obtenidos permiten confirmar una estructura multifactorial con ajuste adecuado de la escala percepción de apoyo social, manteniéndose las dimensiones de percepción de apoyo social de amigos, familia y otras personas significativas propuestos en la versión original de la escala. Se confirma la estructura de un factor general de las variables de percepción de apoyo a la autonomía, compromiso académico e intención de abandono.

Las escalas de percepción de apoyo a la autonomía y compromiso académico se re-especificaron dado que no tuvieron ajustes óptimos; por este motivo se revisaron los índices de modificación, y se incorporaron correlaciones entre los residuos los cuales mejoraron el ajuste de los modelos de medida. Los índices de ajuste de la escala de compromiso académico fueron: $\mathrm{X}^{2}(27)=1702.152, \mathrm{p}<.001 ; \mathrm{CFI}=.819 ; \mathrm{TLI}=.759$; RMSEA = .141 (.135-.147), SRMR = .069. Al introducir los índices de modificación entre los residuos de los ítems 7 y $3(I M=799.179) ; 1$ y $2(I M=348.061)$, se logró un buen ajuste del modelo que se puede observar 
en la tabla 2. Los índices de ajuste de la escala percepción de apoyo a la autonomía fueron: $X^{2}(9)=513.945$, $\mathrm{p}<.001 ; \mathrm{CFI}=.916 ; \mathrm{TLI}=.861 ; \mathrm{RMSEA}=.134(.124-.144), \mathrm{SRMR}=.040$. Mediante la correlación de los errores de los ítems 5 y $6(I M=352.441)$, se logró un buen ajuste el cual se puede observar en la tabla 2.

Tabla 2. Análisis factoriales confirmatorios del apoyo social, apoyo a la autonomía, compromiso e intención de abandono.

\begin{tabular}{|l|c|c|c|c|c|c|}
\hline Variables & $\mathrm{X}^{2}$ & $\mathrm{Df}$ & $\mathrm{RMSEA}$ & $(90 \% \mathrm{CI})$ & $\mathrm{TLI}$ & $\mathrm{CFI}$ \\
\hline Apoyo social & $561.58^{\star *}$ & 51 & .057 & $.052-.061$ & .951 & .962 \\
\hline Apoyo a la autonomía & $187.20^{\star *}$ & 8 & .085 & $.074-.095$ & .944 & .970 \\
\hline Compromiso académico & $553.26^{\star *}$ & 25 & .082 & $.076-.088$ & .918 & .943 \\
\hline Intención de abandono & $7.02^{*}$ & 2 & .028 & $.008-.052$ & .989 & .996 \\
\hline
\end{tabular}

\section{Resultados descriptivos y correlacionales}

En la tabla 3, se presentan los análisis descriptivos; éstos indican que los estudiantes presentaron puntuaciones similares en las dimensiones de percepción de apoyo social $(M=5.66, D E=1.08)$; percepción de apoyo de otros significativos $(M=5.71, D E=1.21)$, percepción de apoyo proveniente de los amigos $(M=$ $5.64, D E=1.34)$ y percepción de apoyo familiar $(M=5.63, D E=1.38)$. En general, presentaron un bajo nivel de intención de abandono $(M=1.72, D E=0.99)$, un nivel adecuado de compromiso académico $(M=5.00$, $D E=1.06)$ y niveles moderados de percepción de autonomía por parte del docente.

Tabla 3. Estadísticos descriptivos y correlaciones entre el apoyo social, apoyo a la autonomía, compromiso académico e intención de abandono.

\begin{tabular}{|c|c|c|c|c|c|c|c|c|}
\hline Variables & $\mathrm{M}$ & $\mathrm{DE}$ & (1) & (2) & (3) & (4) & (5) & (6) \\
\hline (0) Intención de abandono & 1.72 & 0.99 & $-.238^{\star *}$ & $-.201^{* *}$ & $-.186^{\star \star}$ & $-.182^{* *}$ & $-.229^{\star \star}$ & $-.463^{* *}$ \\
\hline (1) Apoyo a la autonomía & 4.85 & 1.16 & & $.279^{\star \star}$ & $.313^{\star \star}$ & $.299^{\star *}$ & $.359^{\star \star}$ & $.433^{\star \star}$ \\
\hline (2) Apoyo familiar & 5.63 & 1.38 & & & $.523^{\star *}$ & $.414^{\star *}$ & $.789^{* *}$ & $.325^{\star *}$ \\
\hline $\begin{array}{l}\text { (3) Apoyo otros } \\
\text { significativos }\end{array}$ & 5.71 & 1.21 & & & & $.653^{\star *}$ & $.864^{\star *}$ & $.341^{\star \star}$ \\
\hline (4) Apoyo de amigos & 5.64 & 1.34 & & & & & $.831^{* *}$ & $.270^{\star *}$ \\
\hline (5) Apoyo social general & 5.66 & 1.08 & & & & & & $.376^{\star *}$ \\
\hline (6) Compromiso académico & 5.00 & 1.06 & & & & & & \\
\hline
\end{tabular}

En la relación de la intención de abandono con el resto de las variables se encontró una correlación inversa con compromiso académico $(r=-.46 ; p<.001)$. El compromiso académico se encuentra relacionado, en mayor medida con la percepción de apoyo a la autonomía $(r=.43, p<.001)$, y con la percepción de apoyo social $(r=.38, p<.001)$. Estos resultados indican que, a mayor nivel de compromiso académico, mayor percepción del apoyo a la autonomía y percepción de apoyo social general; se presenta menor intención de abandono por parte de los estudiantes universitarios. La percepción de apoyo social proveniente de la familia, seguido de otras fuentes significativas, fueron las que presentaron mayor magnitud de asociación con la intención de abandono.

\section{Modelo de ecuaciones estructurales}

El modelo propuesto presentó índices de ajuste satisfactorios: $\mathrm{X}^{2}(424)=3337.99, p<.001 ; \mathrm{CFI}=.931 ; \mathrm{TLI}=$ $.924 ; \mathrm{RMSEA}=.047(.045-.048), \mathrm{SRMR}=.056$. Se detectó un efecto indirecto de la percepción de apoyo a la autonomía $(\beta=-.190, p<.001)$ y de la percepción de apoyo social general $(\beta=-.120, p<.001)$ sobre la intención de abandono en estudiantes universitarios. Además, existe un efecto directo del compromiso académico $(\beta=-.489, p<.001)$ sobre la intención de abandono, siendo esta una mediación de tipo parcial. El modelo analizado predice un $24 \%$ de la variabilidad de la variable de salida. En la figura 1 se presenta el modelo con los resultados encontrados. 


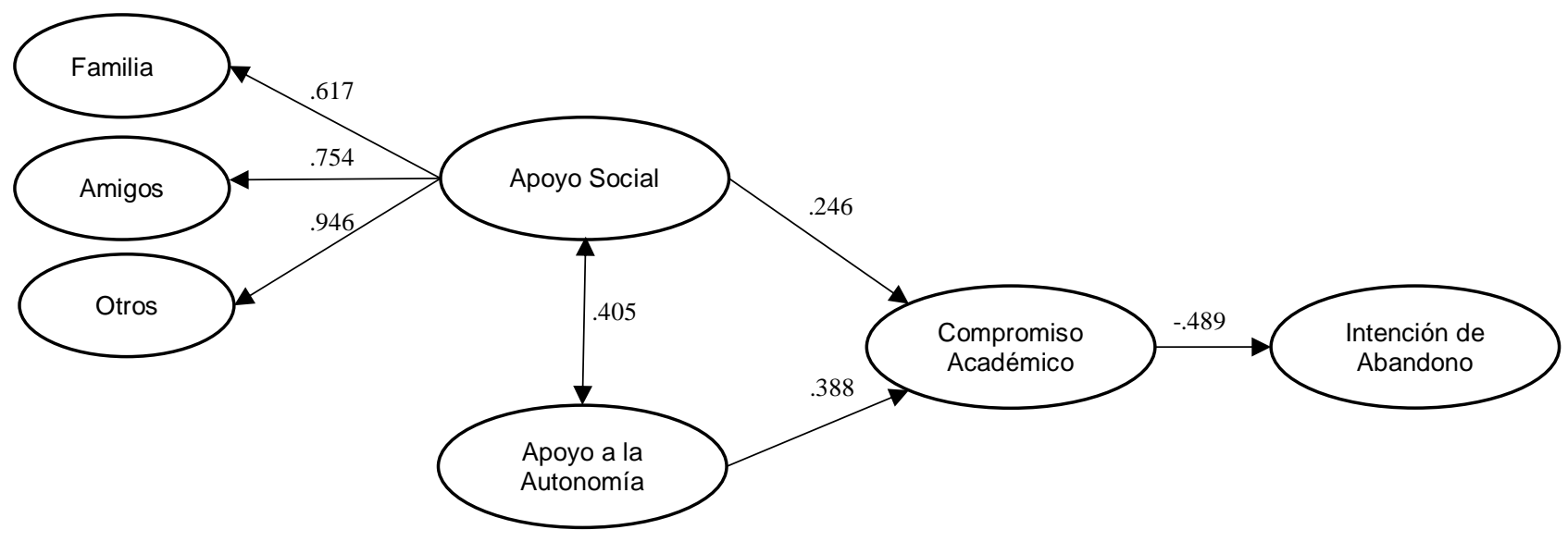

Fig. 1: Modelo de ecuaciones estructurales del apoyo social, apoyo a la autonomía, compromiso académico e intención de abandono de estudiantes de primer año.

\section{DISCUSIÓN}

Este trabajo tuvo como objetivo evaluar la capacidad predictiva de la percepción de apoyo social, la percepción de apoyo a la autonomía y el compromiso académico sobre la intención de abandono de estudiantes universitarios chilenos de primer año. Para esto, se analizó el efecto de mediación del compromiso académico sobre la relación de la percepción de apoyo social, percepción de apoyo a la autonomía con la intención de abandono de los estudios universitarios a través de análisis de ecuaciones estructurales.

Se encontró una correlación inversa entre la intención de abandono y la percepción de apoyo social general, lo que indica que una mayor percepción de apoyo se asocia a disminución en los pensamientos o intenciones de abandonar los estudios. Cuando los estudiantes perciben que pueden acceder a estructuras de soporte social durante el proceso de adaptación a la universidad, el proceso de ajuste al entorno universitario se desarrolla de forma más rápida, beneficiando la integración del estudiante (Páramo et al.,2014). Adicionalmente se identificó que esta asociación presenta mayor magnitud respecto de la percepción de apoyo social proveniente de la familia; existe evidencia de la relevancia que tiene esta percepción de apoyo social proveniente de la familia, siendo un factor significativo para un mejor rendimiento académico en los jóvenes (López-Angulo et. al., 2020). Según esto, la percepción de apoyo social constituye un aspecto que podría ser tomado en cuenta en el diseño de programas intracurriculares de mejora de la docencia universitaria.

Adicionalmente, se encontró una correlación inversa entre la intención de abandono y la percepción de apoyo social proveniente de otras personas significativas. En este caso, la percepción de apoyo proveniente del profesorado, puede tener impacto en la adaptación de los estudiantes en términos académicos, mientras que el apoyo social de la familia puede contribuir a la adaptación personal y emocional de los estudiantes, impactando positivamente en los pensamientos de permanecer (Crede y Niehorster, 2012). Se ha planteado la conveniencia de incluir actividades destinadas a la creación y mantenimiento de redes de apoyo emocionalmente significativas y confiables para los estudiantes durante sus procesos de transición de la secundaria a la universidad, como una estrategia facilite los procesos de adaptación al entorno universitario (Tinajero et al., 2020).

Los hallazgos del presente estudio conducen a investigar la influencia del desarrollo de los vínculos entre los docentes y estudiantes durante el inicio de la carrera universitaria. Estos resultados indican la importancia de basar esta relación en el apoyo y la confianza, promoviendo la sensación de capacidad en los estudiantes para realizar con éxito las tareas que se les presentan (Oriol-Granado et al., 2017) y permitiendo que puedan tomar la iniciativa en su aprendizaje para facilitar el desarrollo de motivaciones auto determinadas (Ryan y Deci, 2000).

La percepción de apoyo a la autonomía de los estudiantes presentó correlación inversa con la intención de abandono, indicando que cuanto mayor es esta percepción de apoyo, menor es la intención de retiro de la carrera; esto puede deberse a la presencia de conductas y sentimientos de dominio de contenidos, y experiencias de aprendizaje, las que pueden fortalecer el proceso de adaptación y la perseverancia durante las actividades académicas (Hernández y Moreno-Murcia, 2017; Oriol-Granado et al., 2017). El modelo de ecuaciones estructurales propuesto indicó la presencia de un efecto directo estadísticamente significativo del compromiso académico en la intención de abandono. El papel del compromiso como mediador permitió 
identificar el efecto indirecto de la percepción de apoyo social y la percepción de apoyo a la autonomía en la intención de abandono. El efecto de la percepción de apoyo a la autonomía fue mayor en el compromiso académico que en la percepción de apoyo social general: Este hallazgo tiene relación con trabajos anteriores que indican la relevancia de la promoción de conductas auto determinadas (Hernández y Moreno-Murcia, 2017; Oriol-Granado et al., 2017), lo que conlleva mayores niveles de energía, involucramiento y concentración en la actividad académica (Schaufeli et al., 2006; Schaufeli et al., 2002).

La identificación del papel predictivo del compromiso académico en la intención de abandono, muestra la relevancia de incluir factores cognitivos y motivacionales en la predicción del abandono en estudiantes universitarios. Este planteamiento ha sido expuesto en otros trabajos, que también valoran la importancia de estas variables en el estudio de los procesos de abandono (Díaz-Mujica et al., 2019). El compromiso académico predice la intención de abandono; cuando el estudiante se encuentra en este estado mental de energía e inspiración, presenta mejor rendimiento académico, emplea en mayor medida sus recursos psicológicos y tiene menos intención de abandonar (Truta et al. 2018; Martínez et al., 2019; Noble y McGrath, 2015).

El modelo analizado predice en un $24 \%$ la intención de abandono de estudiantes universitarios de primer año. Este porcentaje de explicación es aceptable en Ciencias Sociales e indica que aquellos estudiantes que perciban mayor apoyo social y apoyo a su autonomía podrán percibirse más dispuestos para persistir, esforzarse, enorgullecerse, inspirarse, involucrarse y concentrarse en la ejecución de las actividades académicas; lo cual disminuirá su intención de abandono. Este resultado contribuye a la explicación de algunas de las variables que intervienen en la intención de abandono de los estudios universitarios, sobre todo en las áreas más representadas en este estudio (Ingeniería y Tecnología; Ciencias Sociales y Ciencias Médicas y de la Salud).

Esta investigación contribuye a clarificar las relaciones de variables cognitivas y motivacionales en la intención de abandono de estudiantes de primer año. Al mismo tiempo aporta a la escasa evidencia empírica sobre éstas variables en el contexto chileno. Un aspecto relevante es que incluye variables que pueden ser modificadas en la interacción de estudiantes y profesores durante el proceso de enseñanza-aprendizaje. Las implicaciones prácticas derivadas del presente estudio, permiten inferir cuán importante es desarrollar programas que permitan la orientación y la promoción de un proceso de adaptación durante el ingreso a primer año; la focalización de estas estrategias según las características de ingreso de cada estudiante, especialmente en aquellos que presenten indicadores de riesgo (Ferrer-Urbina et al., 2019).

Es conveniente a futuro evaluar el modelo aquí propuesto considerando el tipo de carrera y con otras variables del estudiante (sexo, nota de la enseñanza media). Considerar las puntuaciones de admisión por cada área disciplinar resulta también una opción factible de implementar (Ferrer-Urbina, et al., 2019), todo esto con la finalidad de ampliar la comprensión de estos factores estudiados en la intención del abandono. Se invita a pensar en el desarrollo de investigaciones de tipo longitudinal, puesto que permitirá evaluar cambios en el efecto del compromiso académico, la percepción de apoyo social y de apoyo a la autonomía de los estudiantes en el desarrollo de la carrera universitaria. Estas aportarán al conocimiento de mecanismos que intervienen en el abandono de los estudios universitarios.

\section{CONCLUSIONES}

A partir de los resultados y discusión se pueden establecer las siguientes conclusiones: (1) La percepción de apoyo social y la percepción de apoyo a la autonomía predicen la intención de abandono como efectos indirectos, esta predicción es mediada por el compromiso académico. (2) Las relaciones entre las variables percepción de apoyo social, percepción de apoyo a la autonomía y compromiso académico, constituyen un modelo explicativo de la intención de abandono en estudiantes universitarios chilenos de primer año.

\section{AGRADECIMIENTOS}

Agradecimientos a: (1) Programa CONICYT de Becas Doctorado Nacional Folio 21170795. (2) Proyecto ANID-COVID-1012 "Desarrollo e implementación de procedimientos docentes para facilitar la disposición al aprendizaje en condiciones de distanciamiento físico por pandemia de Covid-19, en asignaturas de primer año universitario con mediano o alto riesgo de fracaso". (3) Proyecto UCO 1808, Laboratorio de Investigación e Innovación Educativa (IDECLAB). (4) Proyecto Fondecyt 1161502.

\section{REFERENCIAS}

Ato, M., López, J. J., y Benavente, A., Un Sistema de Clasificación de los Diseños de Investigación en Psicología, https://doi.org/10.6018/analesps.29.3.178511, An. Psicol., 29(3), 1038-1059 (2013).

Bernardo, A. B., y otros 4 autores, Freshmen Program Withdrawal: Types and Recommendations, https://doi.org/10.3389/fpsyg.2017.01544, Front. Psychol, 8, 1-11 (2017). 
Bordes-Edgar, V., Arredondo, P., Kurpius, S. R., y Rund, J., A Longitudinal Analysis of Latina/o Students' Academic Persistence, https://doi:10.1177/1538192711423318, Int. Educ. Stud., 10(4), 358-368 (2011).

Carmona-Halty, M. A., Schaufeli, W. B., y Salanova, M., The Utrecht Work Engagement Scale for Students (UWES-9S): Factorial Validity, Reliability, and Measurement Invariance in a Chilean Sample of Undergraduate University Students, https://doi:10.3389/fpsyg.2019.01017, Front. Psychol., 10, 1-5 (2019).

Credé, M., y Niehorster, S, Adjustment to College as Measured by the Student Adaptation to College Questionnaire: A Quantitative Review of its Structure and Relationships with Correlates and Consequences, https://doi:10.1007/s10648011-9184-5, Educ Psychol Rev, 24(1), 133-165 (2012).

Díaz-Mujica, A.,y otros 4 autores. Mediación del Ajuste Académico Entre Variables Cognitivo-motivacionales y la Intención de Abandono en Primer año de Universidad. Trabajo presentado en la octava conferencia latinoamericana sobre abandono en la Educación Superior, Panamá (Noviembre, 2018).

Díaz-Mujica, A., y otros 4 autores., Affective and Cognitive Variables Involved in Structural Prediction of University Dropout, https://doi:10.7334/psicothema2019.124, Psicothema, 31(4), 429-436 (2019).

Ferrer-Urbina, R., Karmelic-Pavlov, V., Beck-Fernández, H., y Pinto, R. V., Un Modelo Predictivo de Fracaso/éxito Académico a partir de Indicadores de Ingreso, en Estudiantes de una Universidad Estatal del Norte de Chile, Interc, 44(1), 23-29 (2019).

Gallegos, J. A., Campos, N. A., Canales, K. A., y González, E.N., Factores Determinantes en la Deserción Universitaria. Caso Facultad de Ciencias Económicas y Administrativas de la Universidad Católica de la Santísima Concepción (Chile) https://doi: 10.4067/S0718-50062018000300011, Form Univ, 11(3), 11-18 (2018).

Hernández, E. H y Moreno-Murcia, J., Apoyo a la Autonomía entre Estudiantes, Estrés Percibido y Miedo a la Evaluación Negativa: Relaciones con la Satisfacción con la Vida, Psicol. Conduct., 25(3), 517-528 (2017).

León, J., y Núñez, J. L., Causal Ordering of Basic Psychological Needs and Well-Being, https://doi:10.1007/s11205-0120143-4, Soc Indic Res, 114(2), 243-253 (2013).

López-Angulo, Y., Pérez-Villalobos, M. V., Cobo-Rendón, R. C., y Díaz-Mujica, A. E, Apoyo Social, Sexo y Área del Conocimiento en el Rendimiento Académico Autopercibido de Estudiantes Universitarios Chilenos, https://doi:10.4067/s0718-50062020000s300011, Form. Univ.13(3), 11-18 (2020).

Martínez, I. M., Youssef-Morgan, C. M., Chambel, M. J., y Marques-Pinto, A., Antecedents of Academic Performance of University Students: Academic Engagement and Psychological Capital Resources, https://doi:10.1080/01443410.2019.1623382, J Educ Psychol, 39 (8), 1047-106 (2019).

Matos-Fernández, L., Adaptación de Dos Cuestionarios de Motivación: Autorregulación del Aprendizaje y Clima de Aprendizaje, https://doi:10.26439/persona2009.n012.282, Persona (012), 167-185 (2009).

Noble, T., y McGrath, H., PROSPER: A New Framework for Positive Education, https://doi.org/10.1186/s13612-0150030-2, Psychology of Well-Being, 5 (2), 1-17 (2015).

Oriol-Granado, X., Mendoza-Lira, M., Covarrubias-Apablaza, C. G., y Molina-López, V. M., Emociones Positivas, Apoyo a la Autonomía y Rendimiento de Estudiantes Universitarios: El Papel Mediador del Compromiso Académico y la Autoeficacia, https://doi:10.1016/S1136-1034(17)30043-6, Rev Psicod., 22(1), 45-53 (2017).

Páramo, M., Martínez, Z., Tinajero, C., y Rodríguez, M. S., The Impact of Perceived Social Support in First-year Spanish Sollege Students' Adjustment, J. Intern. Scien Public, 12, 289-300 (2014).

Phillips, L. T., Stephens, N. M., Townsend, S. S., y Goudeau, S., Access is not Enough: Cultural Mismatch Persists to Limit first-generation Students' Opportunities for Achievement Throughout College, https://doi:10.1037/pspi0000234, J Pers Soc Psychol, 119(5), 1112-1131 (2020).

Rodríguez, M. S., Tinajero, C., y Páramo, M. F., Pre-entry Characteristics, Perceived Social Support, Adjustment and Academic Achievement in First-Year Spanish University Students: A Path Model, https://doi:10.1080/00223980.2017.1372351, J Psychol, 151(8), 722-738 (2017).

Ryan, R. M., y Deci, E. L., Self-determination Theory and The Facilitation of Intrinsic Motivation, Social Development, and Well-being, https://doi:10.1037110003-066X.55.1.68, Am Psychol, 55(1), 68-78 (2000).

Salanova, M. S., W.;Martinez, I. y Breso, E., How Obstacles and Facilitators Predict Academic Performance: The Mediating Role of Study Burnout and Engagement, https://doi:10.1080/10615800802609965, Anxiety Stress Coping, 23(1), 53-70 (2010).

Santini, Z. I., y otros 4. The Association Between Social Relationships and Depression: a Systematic Review, https://doi:10.1016/j.jad.2014.12.049, J. Affect. Disord., 175(1), 53-65 (2015).

Schaufeli, W., Bakker, A. B., y Salanova, M., The Measurement of Work Engagement with a Short Questionnaire: A Cross-National Study, https://doi:10.1177/0013164405282471, Educ Psychol Meas, 66(4), 701-716 (2006).

Schaufeli, W., y otros 4 autores, Burnout and Engagement in University Students: A Cross-national Study, https://doi:10.1177/0022022102033005003, J Cross Cult Psychol, 33(5), 464-481 (2002). 
Tinajero, C., Martínez-López, Z., Rodríguez, M. S., y Páramo, M. F., Perceived Social Support as a Predictor of Academic Success in Spanish University Students, https://doi:10.6018/analesps.344141, An. Psicol., 36(1), 134-142 (2020).

Tinto, V., Through the Eyes of Students, https://doi:10.1177/1521025115621917, J Coll Stud Ret, 19(3), 254-269 (2017).

Truta, C., Parv, L., y Topala, I., Academic Engagement and Intention to Drop Out: Levers for Sustainability in Higher Education, https://doi:10.3390/su10124637, Sustainability, 10(12), 1-11 (2018).

Wang, J., y Wang, X., Structural Equation Modeling: Applications Using Mplus: John Wiley y Sons (2019).

Zimet, G. D., Dahlem, N. W., Zimet, S. G., y Farley, G.K., The Multidimensional Scale of Perceived Social Support, https://doi:10.1207/s15327752jpa5201_2, J Pers Assess, 52(1), 30-41 (1988). 
